EGU21-13781, updated on 18 Jan 2022

https://doi.org/10.5194/egusphere-egu21-13781

EGU General Assembly 2021

(c) Author(s) 2022. This work is distributed under

the Creative Commons Attribution 4.0 License.

\title{
The ASKOS experiment for the validation of Aeolus L2A aerosol product
}

Eleni Marinou ${ }^{1}$, Vasslis Amiridis ${ }^{1}$, Ioanna Mavropoulou ${ }^{1}$, Holger Baars ${ }^{2}$, Stelios Kazadzis ${ }^{3}$, Marco Rosoldi ${ }^{4}$, Dragos Ene ${ }^{5}$, Africa Barreto ${ }^{6}$, Stefano Casadio ${ }^{7}$, Cordula Zenk ${ }^{8}$, Jean Sciare ${ }^{9}$, Grisa Mocnik $^{10}$, Konrad Kandler ${ }^{11}$, Jan-Berend Stuut ${ }^{12}$, Sergio Rodrigez ${ }^{13}$, Peter Knipertz ${ }^{14}$, Thomas Rutz ${ }^{15}$, Mika Komppula ${ }^{16}$, Vassiliki Daskalopoulou ${ }^{1}$, George Hloupis ${ }^{17}$, and the ASKOS team ${ }^{*}$

${ }^{1}$ IAASARS, National Observatory of Athens, Athens, Greece (elmarinou@noa.gr)

${ }^{2}$ Leibniz Institute for Tropospheric Research,Leipzig, Germany

${ }^{3}$ Physics and Meteorology Observatorium of Davos, World Radiation Center (PMOD/WRC), Davos, Switzerland

${ }^{4}$ Italian National Research Council Institute of Methodologies for Environmental Analysis, Potenza, Italy

${ }^{5}$ National Institute of R\&D for Optoelectronics, Bucharest, Romanian

${ }^{6}$ Agency Estatal de Meteorologia, Spain

${ }^{7}$ European Space Agency, ESA

${ }^{8}$ GEOMAR Helmholtz Centre for Ocean Research Kiel, Germany

${ }^{9}$ The Cyprus Institute, Nicosia, Cyprus

${ }^{10}$ University of Nova Gorica, Slovenia

${ }^{11}$ Technical University of Darmstadt, Darmstadt, Germany

${ }^{12}$ Royal Netherlands Institute for Sea Research, The Nederlands

${ }^{13}$ Consejo Superior de Investigaciones Científicas, Spain

${ }^{14} \mathrm{Karls}$ ruhe Institute of Technology, Germany

${ }^{15}$ Freie Universität, Berlin, Germany

${ }^{16}$ Finnish Meteorological Institute, Kuopio, Finland

${ }^{17}$ University of West Attica, Athens, Greece

*A full list of authors appears at the end of the abstract

For the in-orbit calibration and validation of the Aeolus products, ESA organized the Aeolus Tropical campaign, which will take place on June-July 2021 at Cape Verde region. During the campaign, Aeolus underfights will be performed with several aircrafts (by DLR, NASA, LATMOS, and the University of Nova Gorica (UNG)) and advanced ground-based instrumentation will be deployed in Mindelo island within ASKOS (https://askos.space.noa.gr/) experiment. ASKOS observations will provide an unprecedented dataset for the aerosol and wind conditions in the region, in order to provide reference values for the Cal/Val of the mission. Apart from the main aerosol Cal/Val objective of ASKOS, the foreseen synergistic activities will provide a wealth of information to address scientific questions posed by the participating groups on dust characterization, transportation and it's impact of radiation and cloud formation.

Here, we report on the status of the ASKOS preparations for the evaluation of the aerosol and cloud product, focusing on the instrumentation requirements and availability, as well as the 
engagement of the scientific community so far. ASKOS will deploy advanced ground-based and airborne remote sensing and in-situ instrumentation, including the full ACTRIS aerosol and cloud remote sensing/in-situ facilities and airborne in-situ sensors to be operated on drones and/or aircrafts. The main ground-based remote sensing instrumentation in Cape Verde will consist of sophisticated lidar systems, including the EVE lidar, a circular polarization system that is tailored to mimic the Aeolus measurement from ground, the multi-wavelength Polly-XT and the WALL-E prototype for detecting particle orientation. The instrumentation will also include sunphotometers such as AERONET-CIMEL, but also polarimeters to advance microphysical retrievals for non-spherical particles such as dust. Cloud remote sensors including a cloud radar and a microwave radiometer will operate in parallel along with meteorological radiosondes. In-situ sensors at surface and onboard UAVs and light aircrafts will be available. ASKOS will be fully supported by several operational modeling simulations for meteorological and atmospheric composition forecasting. ASKOS will remain open to contributions from other communities and research groups and more synergies will be pursued in the future.

ASKOS team: Eleni Marinou (1), Vassilis Amiridis (1), Alexandra Tsekeri (1), Nikos Siomos (1), Peristera Paschou (1), Vassiliki Daskalopoulou (1), Raptis Panos (1), Anna Gialitaki (1), Spyros Metallinos (1), Ioanna Mavropoulou (1), Christos Spyrou (1), Anna Kampouri (1), Eleni Drakaki (1), Ioanna Tsikoudi (1), Maria Tsichla (1), Thanasis Georgiou (1), Antonis Gkikas (1), Proestakis Emmanouil (1), Giorgos Papangelis (1), Sotiris Mallios (1), Stavros Solomos (1), Stavroupa Papatheochari (1), Alexandros Alexiou (1), Holger Baars (2), Annett Scupin (2), Dietrich Althausen (2), Ronny Engelmann (2), Stelios Kazadzis (3), Angelos Karanikolas (3), Marco Rosoldi (4), Lucia Mona (4), Dragos Ene (5), Doina Nicolae (5), Livio Belegante (5), Flori Toanca (5), Mariana Adam (5), Africa Barreto (6), Emilio Cuevas (6), Natalia Prats (6), Stefano Casadio (7), Thorsten Fehr (7), Cordula Zenk (8), Elizandro Rodrigues (8), Silva Eder (8), Pericles Silva (8), Jean Sciare (9), Maria Kezoudi (9), Panos Vouterakos (9), Maximilien Desservettaz (9), Florin Unga (9), Panayiota Antoniou (9), Christos Keleshis (9), Grisa Mocnik (10),Konrad Kandler (11), Arya Sree (11), Jan-Berend Stuut (12), Sergio Rodrigues (13), Peter Knippertz (14), Andreas Fink (14), Maurus Borne (14), Thomas Rutz 15), Jonas Lehmke (15), Lena Jänicke (15), Mika Komppula (16), Georgios Hloupis (17), Ilias Makrakis (17), Joseph Ulanowski (18), Josef Gasteiger (19), Volker Freudenthaler (20), Bruno Faria (21), Katie Read (22), Luis Neves (22), Claire Ryder (23), Gaël Dubois (24), Phillipe Golub (24). Affiliations: (1) IAASARS, National Observatory of Athens, Athens, Greece; (2) Leibniz Institute for Tropospheric Research, Leipzig, Germany; (3) Physics and Meteorology Observatorium of Davos, World Radiation Center (PMOD/WRC), Davos, Switzerland; (4) Italian National Research Council Institute of Methodologies for Environmental Analysis, Potenza, Italy; (5)National Institute of R\&D for Optoelectronics, Bucharest, Romanian; (6) Agency Estatal de Meteorologia, Spain; (7) European Space Agency, ESA; (8) GEOMAR Helmholtz Centre for Ocean Research Kiel, Germany; (9) The Cyprus Institute, Nicosia, Cyprus; (10) University of Nova Gorica, Slovenia; (11) Technical University of Darmstadt, Darmstadt, Germany; (12) Royal Netherlands Institute for Sea Research, The Netherlands; (13) Consejo Superior de Investigaciones Científicas, Spain; (14) Karlsruhe Institute of Technology, Germany; (15) Freie Universität, Berlin, Germany; (16) Finnish Meteorological Institute, Kuopio, Finland; (17) University of West Attica, Athens, Greece; (18) University of Hertfordshire, Hertfordshire, United Kingdom; (19) University of Vienna, Vienna, Austria; (20) Ludwig Maximilian University of Munich, Munich, Germany; (21) National Institute of Meteorology and Geophysics, Cape Verde; (22) Cape Verde Atmospheric Observatory, Cape Verde; (23) University of Reading, Reading, UK; (24) Lille University, Lille, France. 